\title{
Short Term Prediction of Coal Mine Methane Concentration with Chaos PSO-RBFNN Model in Underground Coal Mines
}

\author{
Yue Geng \\ School of Mechanical Electronic and Information Engineering, Chia University of Mining and \\ Technology (Beijing), Beijing 100083, China
}

13011197054@163.com

Keywords: Coal mine gas concentration prediction, Chaotic analysis, PSO, RBFNN.

\begin{abstract}
Gas disaster is the serious threat to coal mine safety, the accurate prediction of coal mine methane (CMM) is one effective method avoiding the hazard occurrence. This paper first confirmed the chaotic characteristic of CMM sequence and calculated the delay time and embedding dimension. Combined chaotic sequential phase space reconstruction and the particle swarms optimized RBF neural network (PSO-RBFNN), build a new coupled model. The Chaotic reconstructed Time-series input PSO-RBF neural network model was proposed and highlighted its advantages by comparing the other three conventional models, Time-series input RBFNN (T-RBFNN), Chaotic reconstructed Time-series input RBFNN (CT-RBFNN) and PSO-RBFNN. The performance rank was CT-PSORBFNN, CT-RBFNN, T-PSO-RBFNN, T-RBFNN.
\end{abstract}

\section{Introduction}

In China, deep underground coal mines with severe working conditions are not rare. Accompanied with different hazards, the coal mine safety need to be pay more attention. Among those underground accidents, coal mine gas hazard always accounts the highest proportion and may induce coal mine gas fire, even explosion. Under the above background, the research and scientific efforts focus on coal mine gas forecasting and prediction have never been stopped. Liu and Qiao[1] analyzed the relationship of the main parameters and build mechanical model by using numerical simulation. Wu el. [2] build a grey system based model, transmit the historical monitor data into grey data and analyzed association degree, discriminating distinction for the final coal mine gas prediction. CMM (Coal Mine Methane) hazards are always effected by several variables, the geological method from [1] could be used to analyze the complex relationship and successfully applied to some practical situations. However, only by the premise of several hypotheses can the above conventional method by used in limited numerical simulation. The biggest problem of grey system model is powerless nonlinear analysis. For the better research on the nonlinear dynamic CMM system, Chaos theory [3] and artificial neural networks (ANN) [4-8] had been applied. Cheng el. [3] applied chaotic time series analysis method and built a chaotic model to forecast the methane concentration. Zhu el. [4] proposed a typical three-layer structure back-propagation neural network to estimate the gas emission. Similarly, Wei el. [5] trained BPNN gradient descending. However, the models from [4-5] may be computed expensively and also the gradient descent training may bring the optimum to the local maxima. Realizing those problems, in [6], Zhu and Zhang applied the genetic algorithm to globally search the parameters of NN for avoiding the local maxima. Gao el. [7] proposed Wavelet RBFNN to predict the CMM concentration and speed up the training process.

For the purpose to avoid the CMM related disasters by accuracy forecasting and improve the conventional empirical methods, this paper combined Chaos and artificial neural networks and determined the parameters of ANN by the global fast search PSO algorithm.

\section{Chaotic phase space reconstruction}

Thanks to the contribution of Takens embedding theorem, the nonlinear dynamical system (like CMM system) can be analyzed via reconstruct attractors in phase space. The reconstruct attractors are diffeomorphic and topological equivalent with the original dynamic system, which can be 
obtained by the reconstructed the observation time series. The reconstruct time series is the low dimensional projection of the underlying trajectory, unfolding the observation sequence can form a pseudo-state space and present more evolution information and feature by those reconstructed points [9]. In this paper, Cao method and the average mutual information (AMI) [10] were used to determine the two crucial parameters: embedding dimension $\mathrm{m}$ and delay time $\tau$ of the observation CMM concentration time series and form the phase space reconstruction. The Wolf method [10] was applied to judge the chaos discrimination of the CMM system in this paper. The computed positive maximum Lyapunov exponent represented the chaotic characteristic.

\section{The Partial Swarms Optimized Radial Basis Function Neural Network (PSO-FNN)}

RBFNN is a three-layer structure feedforward neural network, the low dimensional inputs can be nonlinear transformed to high dimension by the hidden activate RBFs. The transformation act as local high dimensional phase space and turns to be high dimensional classified. The topological structure shown as follow:

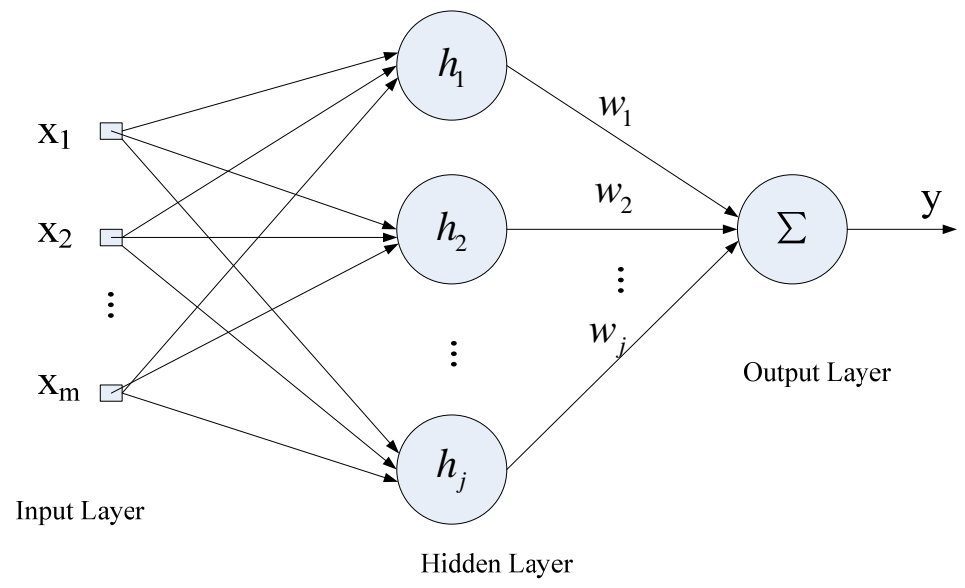

Fig 1. Radial Basis Function Neural Network Structure

In this paper, Gaussian RBFs were chosen to be the hidden layer activation and RMS error was used to train the model. Compared to the global searching model BPNN, the local search model RBFNN is in superiority on simple structure, fast training and cheap computing. However, the training process of one typical RBFNN only search locally and update the parameters (linking weight $w$, RBF centers $c$ and variance $\sigma$ ) empirically. To improve this, PSO algorithm was used in this paper. As a globally random searching intelligent algorithm, PSO can improve the limitation of RBFNN by treat the crucial parameters as the particle swarms. After random initialization, each particle owns velocity and position information. Find the best information of individual and population in each step and replace the best information constantly, the searching finally convergence and the optimum parameters obtained.

\section{Real CMM data simulation}

The real CMM data used in this paper comes from the underground coal mine monitoring system of Xuan Dong Cola Mine. And the relevant time span is the first season of 2013 with one-minute sampling time. The whole process flow chart shown as follow: 


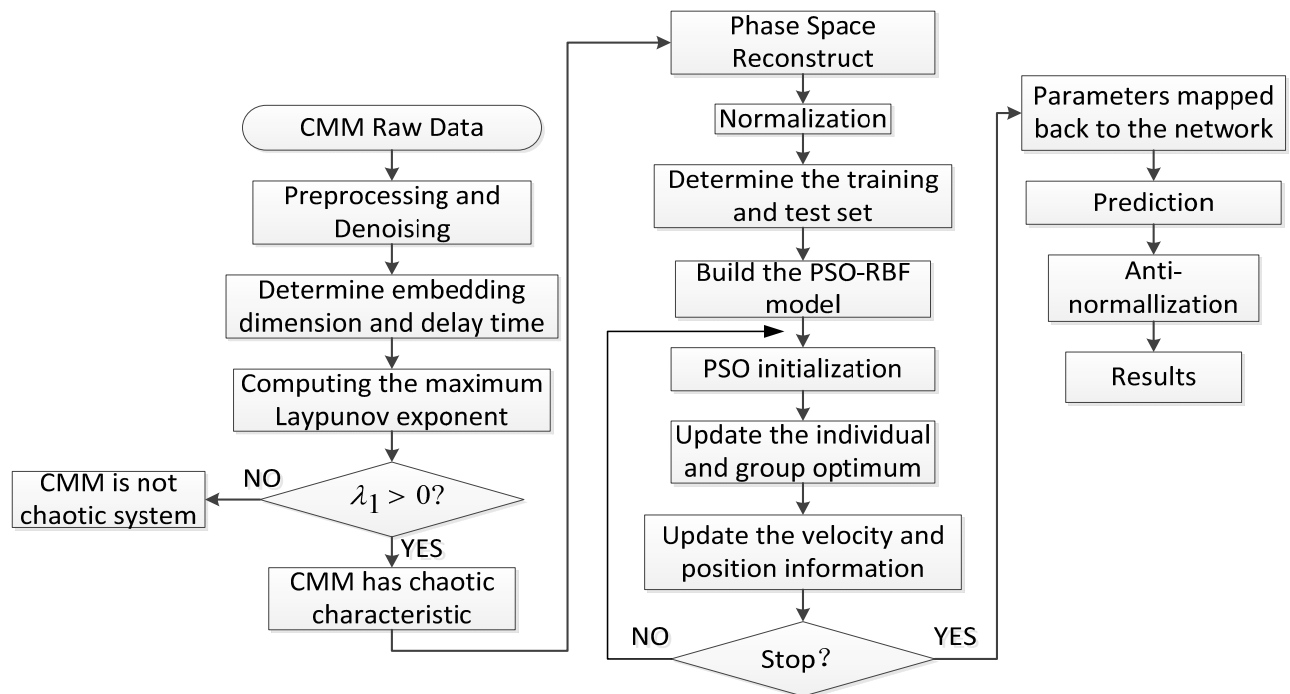

Fig. 2 CT-PSO-RBFNN Prediction Flow Chart

Due to the complex production environment, the raw data may be noisy and deficient which caused by the environment and human factor. Mean value replacement and soft threshold wavelet [7] were used to preprocess and noise-reduce the raw data, which were shown as Figure 3. The embedding dimension and delay time were determined as $m=3$ and $\tau=9$ with Cao method and AMI algorithm, the maximum Lyapunov exponent calculated by wolf algorithm is $\lambda_{1}=0.019$ which proved the chaotic characteristic of CMM system. The reconstructed phase space shown as Figure 4. Normalized the raw data and chose the front 6880 points as the training set while the others as the testing set. The initial parameters of PSO algorithm shown in Table 1.
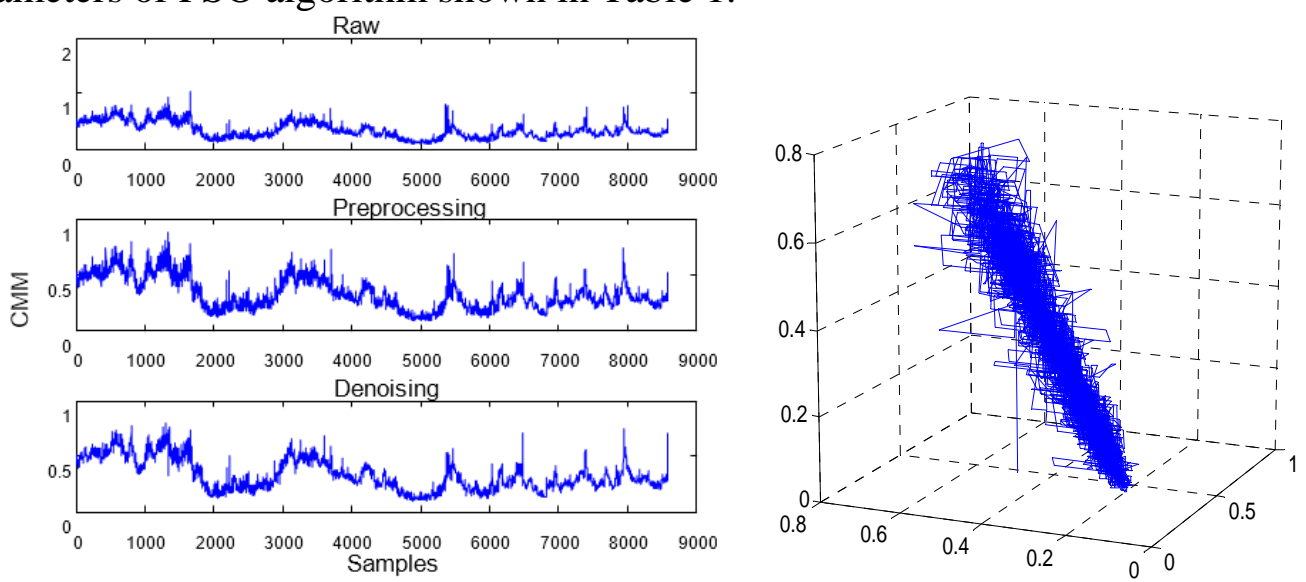

Fig. 3 Data Preprocessing Results

Fig. 4 Reconstruction 3D Display

Table 1 PSO Initialization

\begin{tabular}{ccccc}
\hline Population & Inertia weight & Particle dimension & Learning rate & Iteration \\
\hline$m=20$ & $\mathrm{w}_{1}=0.1$ & $n=25$ & $l_{1}=l_{2}=2$ & 250 \\
\hline
\end{tabular}

The 3-5-1 structure of RBFNN was defined with three delayed reconstructed time series inputs, five hidden layer nodes and one output predicted CMM concentration. To highlight the advantages of the proposed CT-PSO-RBFNN, T-RBFNN, CT-RBFNN and PSO-RBFNN were compared with CT-PSO-RBFNN in the next part. The CMM prediction results and error of the proposed model and the relevant error were shown as Figure 5 and 6, the relevant prediction error ratio in Table 2. 


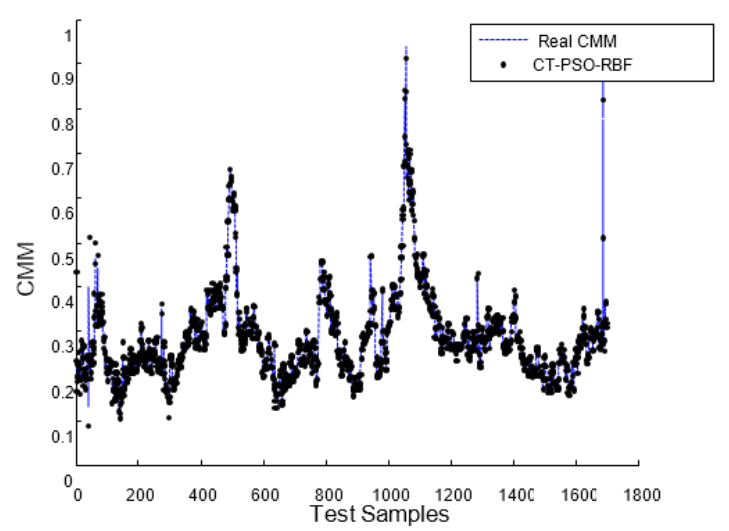

Fig.5 CT-PSO-RBFNN Prediction Result

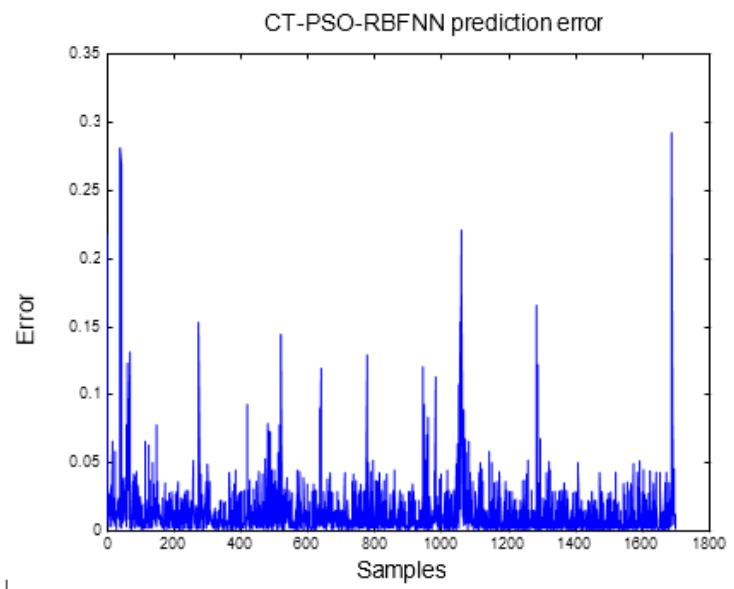

Fig.6 CT-PSO-RBFNN Prediction Error

Table 2 Prediction Error Ratio of CT-PSO-RBFNN

\begin{tabular}{cccc}
\hline Data number below 10\% & Data number beyond 20\% & Max error & Min error \\
\hline 1590 & 7 & 0.29 & $2.94 \times 10^{-8}$ \\
\hline
\end{tabular}

The contrast of four models CMM predictions and the relevant errors were shown as Figure 7-8 and Table 3.

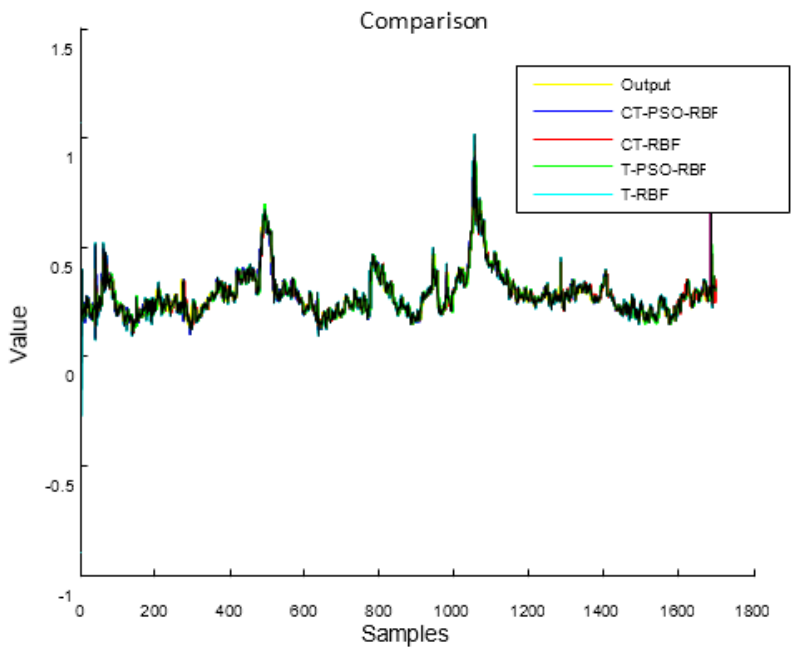

Fig.7 Contrast of Four Models

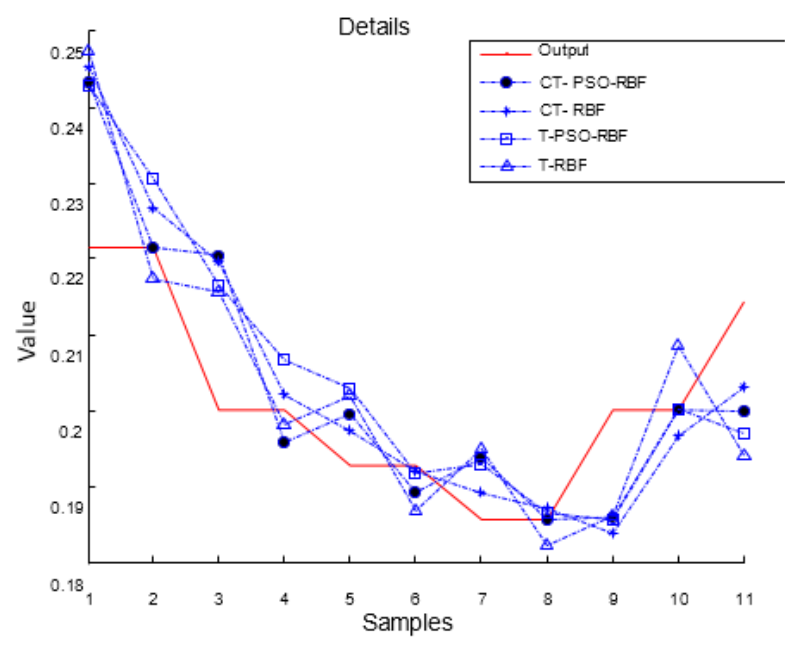

Fig.8 Local Contrast Detail

Table 3 Contrast of Four Model Prediction Errors

\begin{tabular}{ccccc}
\hline & CT-PSO-RBFNN & CT-RBFNN & T-PSO-RBFNN & T- RBFNN \\
\hline Mean AE & $\mathbf{0 . 0 1 3 4}$ & 0.0141 & 0.0157 & 0.0188 \\
MAE & $\mathbf{0 . 3 1 0 8}$ & 0.6802 & 0.6145 & 1.1062 \\
SD & $\mathbf{0 . 0 9 8 6}$ & 0.0992 & 0.0982 & 0.1074 \\
RMSE & $\mathbf{0 . 0 2 6 5}$ & 0.0344 & 0.0308 & 0.0502 \\
\hline
\end{tabular}

\section{Conclusion}

The results observed from Fig. 5-6 and Table 2 proved that the proposed CT-PSO-RBFNN model can predict the real CMM concentration accurately with satisfactory results and minor error. The comparisons of the four different NN models from Fig. 7-8 and Table 3 illustrated the model performances and the relevant rank could be CT-PSO-RBFNN $>$ T-PSO-RBFNN $>$ CT-RBFNN $>$ TRBFNN. The result can be explained from the limitation of RBFNN and the observation CMM concentration lack of information. Chaotic reconstruction applied in this paper to unfold the underlying trajectory and offer more information of the CMM dynamic system. PSO algorithm was used to globally search the parameters of NN and forbidden the local optimum. The simulation indicated the proposed CT-PSO-RBFNN model can forecast the real coal mine gas concentration with relatively accuracy and have an impact on coal mine safety. 


\section{References}

[1]. Liu Junjie, Qiao Deqing, et al. Some aspects on the gas outburst/explosion hazard in coal mine of China. Journal of China coal society. Vol. 31(2006) N0 .1, p.58-62.

[2]. Wu Aiyou, Tian Yunli, Song Yi, et al. Application of the grey system theary for predicting the amount of mine gas emission in coal mine. Journal of China coal society. Vol. 30(2005) N0 .5, p.489-592.

[3]. Cheng Jian, Bai Jingyi, Qian Jiansheng, et al. Short-term forecasting method of coalmine gas concentration based on chaotic time series. Journal of China university of mining \& technology. Vol.37(2008) No.2, p231-235.

[4]. Zhu Hongqing, Chang Wenjie, Zhang Bin, et al. Different source gas emission prediction model of working face based on BP artificial neural network and its application. Journal of China coal society. Vol.32(2007) No.5, p504-508.

[5]. Wei Jianping, Hao Tianxuan, Liu Mingju, et al. BP model of gas content prediction based on quantitative assessment of geological structure complexity. Journal of China coal society. Vol. 34(2009) N0 .8, p.1090-1094.

[6]. Zhu Yu, Zhang Hong, Su Cheng, et al. Coal and gas outburst forecasting based on immune genetic algorithm. Journal of China coal society. Vol. 38(2009) N0 .1, p.125-130.

[7]. Gao Li, Hu Yanjun, Yu Hongzhen, et al. Prediction of gas emission time series based on W-RBF. Journal of China coal society. Vol. 33(2008) N0 .1, p.67-70.

[8]. Fu Hua, Jiang Wei, Shan Xinxin, et al. Study on coupling algorithm on coal mine gas emission forecast model. Journal of China coal society. Vol. 37(2012) N0 .4, p.654-658.

[9]. Ioannis V, Dimitris K. Non-uniform state space reconstruction and coupling detection. Phys. Rev E82,016207(2010), p.1-16

[10]. Zhang Shu-Qing, Jia Jian Gao Min, et al. Study on the parameters determination for reconstructing phase-space in chaos time series. ACTA PHYSICA SINICA. Vol.59(2010) No.3, p.1576-1582. 